\section{P226 TO WORRY OR NOT TO WORRY? THAT IS THE PROBLEM}

${ }^{1}$ Enrica Manca*, ${ }^{2}$ Carmela De Meco, ${ }^{1}$ Agostino Petraccaro, ${ }^{2}$ Irene Rutigliano, ${ }^{1}$ Rosa Canestrale, ${ }^{1}$ Sofia Siena, ${ }^{1}$ Rossella Giorgio, ${ }^{2}$ Michele Sacco, ${ }^{2}$ Massimo PettoelloMantovani. ${ }^{1}$ Residency Program in Pediatrics, University of Foggia, Foggia, Italy; ${ }^{2}$ Department of Pediatrics, Pediatric Unit, 'Casa Sollievo della Sofferenza' Scientific Institute, University of Foggia, San Giovanni Rotondo, Foggia, Italy

10.1136/archdischild-2019-epa.576

Benign neonatal pustolosis (BNP) comprehend a group of clinical diseases characterized by transient pustules or vescico-pustular lesions on newborn skin. These are asymptomatic and self-limiting conditions including benign cephalic pustolosis (BCP).

A 40 days old girl was conducted to our Pediatric Unit for the appearance of multiple vescico-pustular lesions with serous sterile content on her forehead. She was born at term, had a regular perinatal period and never reported cutaneous problems. No lotions or creams for and after her baths were used and no direct contact to sunlight was described. At the time of consultation, she was in excellent health conditions. On suspicion of BCP, we did not prescribe exams or local and/or systemic treatments but indicated a strict follow-up with clinical revaluation after 3 days. At follow-up, the infant did not have clinical problems and her pustular lesions had begun to disappear. After 7 days, all pustules had completely disappeared without leaving any scar.

Dermatosis that occur during the neonatal period can be infectious or sterile, such as BNP. Frequently, BNP are secondary to a physiological skin response or to environmental factors. They are benign, self-limited, asymptomatic cutaneous conditions that present during the first days of life. Their diagnosis is clinical but, sometimes, can require some investigations, principally non-invasive, to exclude more severe diseases. BNP include erythema toxicum neonatorum, transient neonatal pustular melanosis and BCP. BCP was first described by Aractingi in 1991. There is no consensus about its prevalence, which is estimated between $10 \%$ and $60 \%$. Its presentation is asymptomatic and self-limiting and is characterized by numerous papules and pustules located on the face and scalp with onset between 5 days and approximately 3 weeks of age of the newborn. Numerous studies evaluated the possible role of Malassezia in the etiopathogenesis of BCP. Nevertheless, this correlation has not been demonstrated so far.

In conclusion, the presence of pustules in newborns is always a reason of concern for parents and doctors, since neonatal skin is more vulnerable to bacterial, viral and fungal infections. These lesions can be a real challenge for clinicians who have to recognize serious diseases requiring hospitalisation from benign transient conditions, avoiding superfluous exams, treatments and worries.

\section{P227 THERE IS MORE TO SKIN THAN MEETS THE EYE: CHILDHOOD PSORIASIS AND METABOLIC SYNDROME. EARLY MORBIDITY REQUIRES EARLY INTERVENTION}

Doireann Roantree*. University of Limerick Graduate Entry Medical School, Limerick , Ireland. He Charles Centre for Dermatology, University Hospital Limerick, Limerick , Ireland

10.1136/archdischild-2019-epa.577

Introduction One third of psoriasis cases have a paediatric onset. In Ireland, an estimated 20,000 children have psoriasis. In adults, the condition has been associated with comorbidities including metabolic syndrome (MetS) leading to premature cardiovascular disease and early mortality. If a similar relationship between paediatric psoriasis and MetS exists, then early detection and monitoring is vital to improve morbidity and mortality. Aims Ascertain the burden of disease of severe psoriasis in children being treated in our department. Evaluate current literature available on the relationship between childhood psoriasis and MetS.

Methods A search of patient records for the terms 'biologics' and 'methotrexate' was undertaken over a 6-month study period from November 2017-April 2018. In addition, a search of the electronic databases PubMed, MEDLINE, EMBASE and Google Scholar from 1966 to July 2018 was undertaken. The inclusion criteria were children between $0-18$ years of age with psoriasis.

Results 71 patients were taking methotrexate. Fourteen were paediatric and seven of the fourteen had psoriasis - $10 \%$ of all patients on methotrexate were children with psoriasis. In the literature search, eighteen papers met the criteria. Six papers assessed the presence of MetS in children with psoriasis with positive findings in all six papers. In separate studies, a positive association was found between dyslipidaemia, hypertension, abdominal obesity and childhood psoriasis.

Conclusion In our centre $10 \%$ of all patients taking methotrexate were children with psoriasis, indicating a significant burden of disease. Ireland's rising obesity rates will trigger new, or exacerbate existing, cases of psoriasis. Our findings support increasing the number of paediatric dermatologists to ensure children with psoriasis are diagnosed and treated promptly to reduce the risk of metabolic syndrome with its cardiovascular and metabolic manifestations.

\section{P228 AN AUDIT OF EMERGENCY NEONATAL AND PAEDIATRIC TRANSFERS FROM SLIGO UNIVERSITY (SUH) TO TERTIARY CENTRES OVER A TWO-YEAR PERIOD}

Samy Allawendy*, Emmanuel Osakwe, Dara Gallagher, Kieran Cunningham, Wouter Jonkers. SUH, Sligo, Ireland

\subsection{6/archdischild-2019-epa.578}

An Audit of Emergency Neonatal and Paediatric Transfers from Sligo University (SUH) to tertiary centres over a twoyear period.

Aims To audit the pathway of emergency transfers from Sligo University Hospital to tertiary centres in terms of indication for transfer, mode of transfer and efficiency of practices, and to describe the demographics of patients requiring transfer.

Methods We retrospectively audited cases transferred during the last two years (2017 and 2018). A simple data collection tool was constructed for the purposes of this audit and it facilitated the analysis and presentation of results.

Results A total of 57 patients required emergency transfer to a tertiary centre in 2017 and 2018 (27 and 30 respectively). $54 \%$ of which were neonatal and $46 \%$ were paediatric. $80 \%$ of neonatal patients were transferred by NNTP while $92 \%$ of paediatric patients were transferred by SUH staff. The majority of patients were accepted by Crumlin (OLCHC) (27\% of neonatal \& 61\% of paediatric transfers). Secondly, Children's University Hospital Temple St (CUH) (21\% of neonatal \& $19 \%$ of paediatric transfers). The most common cause for 
transfer was prematurity for the neonate, and respiratory failure for paediatrics.

Conclusion Optimizing and aligning practices between sending and receiving hospitals may improve inter-hospital handover efficiency and patient safety. Accurate recording of transfer data may be used to advocate expansion of the transfer service.

\section{P229 NAPKIN PSORIASIS-A REPORT OF TWO CASES}

Iva Topalusic*, Zdenka Plesa Premilovac, Nives Pustišek, Arnes Rešić. Children's Hospital Zagreb, Zagreb, Croatia

\subsection{6/archdischild-2019-epa.579}

Introduction Psoriasis is a T-cell mediated chronic inflammatory disorder of the skin. There are three age groups of pediatric psoriasis: infantile psoriasis, early onset psoriasis and pediatric psoriasis with psoriatic arthritis. Upper respiratory infection is the most common trigger of infantile psoriasis. We report two patients of early, Napkin psoriasis.

Case 1. A 5-months old female baby was reffered to our Emergency Department due to annular erythematous plaques in forehead, postauricular area, elbows, knees, back and diaper area. Due to suspected infection, ceftriaxon was introduced into therapy. Also, dexamthasone, mupirocin, and clotrimazole were localy applied. As therapy didn't show any clinical effect, punch biopsy was done and showed psoriasis vulgaris. Local therapy with aclomethasondipropionate was continued with good clinical response. A child was followed up during one year and no relapse was recorded.

Case 2. Nearly one-year old female baby was reffered to our Emergency Department after one week of antibiotical treatment of periorbital rash which was spread to the forehead, postauricular area, elbows, knees, back and diaper area. Napkin psoriasis was suspected and dexamethasone was localy applied. As it showed good clinal response, we concluded biopsy was not necessary. None of the children had signs of respiratory infection.

Conclusion Differential diagnosis of diaper dermatitis includes Napkin psoriasis, candidal diaper dermatitis and allergic contact dermatitis. In almost one third of all cases, psoriasis begins in childhood. Infants with psoriasis usually present with a diaper rash that is unresponsive to irritant diaper dermatitis treatment. Therefore all patients with diaper dermatitis should be properly evaluated and followed up.

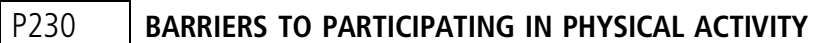 AND EXERCISE IN CHILDREN WITH TYPE 1 DIABETES MELLITUS (T1DM)}

Julie Evers, Madalene Khalil, Niall Dalton*, Orla Neylon, Paul Scully, Clodagh O'Gorman. University Hospital Limerick, Limerick, Ireland

\subsection{6/archdischild-2019-epa.580}

Aims Exercise and physical activity is an important component of a healthy lifestyle in all individuals with T1DM. However, despite efforts to promote an active lifestyle, a significant proportion of children with diabetes remain inactive and do not reach the recommended guidelines for exercise and physical activity. The aim of the current study was to examine barriers to undertaking physical activity in young people with T1DM.
Methods The study was a regional level observational clinical one within the University Hospital Limerick T1DM outpatient clinic, focusing on the perceived barriers to exercise and physical activity using the modified Barriers to physical activity in patient with Diabetes type 1 (BAPAD1) questionnaire. Questionnaires were completed between June and August 2018 and analysed using SPSS statistical software.

Results 55 children completed the survey. 55\% of respondents were female and age range was from 5-17 years, with all respondents been diagnosed a minimum of 2 years previously. $5 \%$ of respondents felt that having diabetes was a barrier to undertaking physical activity. Half of respondents stated that the risk of hypoglycaemia would be likely or very likely to be a barrier to undertaking physical activity. 29\% of respondents said that loss of control of their diabetes was not a barrier to undertaking physical activity. About 30\% of respondents felt that the risk of hyperglycaemia was a barrier to undertaking physical activity. $15 \%$ of respondents felt that their school schedule was a barrier to undertaking physical activity.

Conclusion The results from this study show that a high proportion of children felt that having T1DM was not a barrier to physical activity. The risk of hypoglycaemia was a significant barrier to activity. Future work should focus on addressing these barriers in more detail and the creation of guidance documents regarding overcoming such barriers.

\section{P231 EXPLORING PARENTAL KNOWLEDGE AND INFORMATION SOURCES PRIOR TO TYPE 1 DIABETES DIAGNOSIS TO INFORM FUTURE HEALTH PROMOTION CAMPAIGNS}

${ }^{1}$ Becky Hackett*, ${ }^{2}$ Edna Roche. ${ }^{1}$ The University of Dublin, Trinity College Dublin, Dublin, Ireland; '2Discipline of Paediatrics, The University of Dublin, Trinity College Dublin, Dublin, Ireland

\subsection{6/archdischild-2019-epa.581}

Introduction The diagnosis of Type 1 Diabetes (T1D) can be prolonged with delayed recognition of symptoms and delayed help seeking. Delayed diagnosis can result in the development of Diabetic ketoaciodosis (DKA), a potentially fatal metabolic decompensation resulting in a lasting trajectory of poor glycaemic control with increased risk of diabetes related complications. Health promotion campaigns designed to increase awareness of Type 1 diabetes have prompted earlier diagnosis and reduced the number of children and adolescents presenting in DKA at diagnosis in the majority of populations. Targeting of the media used in such health promotion campaigns would be anticipated to increase the effectiveness.

Aim To explore parental knowledge of T1D before their child's diagnosis, the pathway to diagnosis and parents most valued sources of health information to inform future health promotion campaigns.

Methods Parents of young children diagnosed with T1D attending a national patient and family support organisation event, completed a questionnaire exploring diabetes knowledge, symptoms, pathway to diagnosis and information sources.

Results Parents of twenty-five children participated. Median age at diagnosis was 5.0 (range 0.6-9.3) years. Median time since diagnosis was $1.8(0.1-10.25)$ years. Parents reported the classic T1D symptoms and suspected T1D in 56\% of cases. Pre-diagnosis $80 \%$ knew someone with T1D. Median duration 